\title{
Interim analysis of a multicenter registry study of COVID-19 patients with inflammatory bowel disease in Japan (J-COSMOS)
}

\author{
Hiroshi Nakase $^{1}$ (D) Yuki Hayashi $^{1} \cdot$ Daisuke Hirayama $^{1}$ ' Takayuki Matsumoto $^{2}$. \\ Minoru Matsuura $^{3} \cdot$ Hideki Iijima $^{4} \cdot$ Katsuyoshi Matsuoka $^{5} \cdot$ Naoki Ohmiya $^{6}$. \\ Shunji Ishihara ${ }^{7}$ Fumihito Hirai $^{8}$ - Daiki Abukawa ${ }^{9}$ Tadakazu Hisamatsu ${ }^{3}$. \\ J-COSMOS group
}

Received: 17 December 2021 / Accepted: 10 January 2022 / Published online: 28 January 2022

(C) The Author(s) 2022

\begin{abstract}
Background The spread of coronavirus disease 2019 (COVID-19) had a major impact on the health of people worldwide. The clinical background and clinical course of inflammatory bowel disease (IBD) among Japanese patients with COVID-19 remains unclear.

Methods This study is an observational cohort of Japanese IBD patients diagnosed with COVID-19. Data on age, sex, IBD (classification, treatment, and activity), COVID-19
\end{abstract}

Members of the J-COSMOS group are listed under

Acknowledgements section.

Hiroshi Nakase

hiropynakase@gmail.com

1 Department of Gastroenterology and Hepatology, Sapporo Medical University School of Medicine, S-1, W-16, Chuoku, Sapporo, Hokkaido 060-8543, Japan

2 Division of Gastroenterology, Department of Medicine, Iwate Medical University, Morioka, Japan

3 Department of Gastroenterology and Hepatology, Kyorin University School of Medicine, Tokyo, Japan

4 Department of Gastroenterology and Hepatology, Osaka University Graduate School of Medicine, Osaka, Japan

5 Department of Gastroenterology and Hepatology, Toho University Sakura Medical Center, Chiba, Japan

6 Department of Gastroenterology, Fujita Health University School of Medicine, Aichi, Japan

7 Department of Gastroenterology, Faculty of Medicine, Shimane University, Izumo, Japan

8 Department of Gastroenterology and Medicine, Fukuoka University Faculty of Medicine, Fukuoka, Japan

9 Department of Gastroenterology and Hepatology, Miyagi Children's Hospital, Sendai, Japan symptoms and severity, and treatment of COVID-19 were analyzed.

Results From 72 participating facilities in Japan, 187 patients were registered from June 2020 to October 2021. The estimated incidence of COVID19 in Japanese IBD patients was $0.61 \%$. The majority of IBD patients with COVID-19 (73\%) were in clinical remission. According to the WHO classification regarding COVID-19 severity, 93\% $(172 / 184)$ of IBD patients had non-severe episodes, while $7 \%(12 / 184)$ were severe cases including serious conditions. $90.9 \%$ (165/187) of IBD patients with COVID-19 had no change in IBD disease activity. A logistic regression analysis stepwise method revealed that older age, higher body mass index (BMI), and steroid use were independent risk factors for COVID-19 severity. Six of nine patients who had COVID-19 after vaccination were receiving antitumor necrosis factor (TNF)- $\alpha$ antibodies.

Conclusion Age, BMI and steroid use were associated with COVID-19 severity in Japanese IBD patients.

Keywords COVID-19 · SARS-CoV-2 · Inflammatory bowel disease $\cdot$ Steroid $\cdot$ Anti-TNF- $\alpha$ antibodies

\section{Introduction}

The rapid spread of severe acute respiratory syndrome coronavirus-2 (SARS-CoV-2) and the resulting coronavirus disease (COVID-19) have impacted patients and healthcare workers in clinical settings [1-3]. The older group of people have a higher incidence of contracting severe COVID-19 [4] since they have weakened immune functions, which is one of the risk factors of COVID-19 [5, 6]. However, in Japan, the impact of COVID-19 on patients 
with various immune diseases and the factors contributing the severity of COVID-19 have yet to be elucidated.

Despite the focus on respiratory symptoms during the diagnosis of COVID-19, gastrointestinal (GI) symptoms including vomiting, diarrhea, and abdominal pain have been reported among COVID-19 patients [7-11], which indicated the involvement of SARS-CoV-2 in intestinal inflammation $[12,13]$.

Chronic intestinal inflammation is among the indications of inflammatory bowel disease (IBD), with an increasing prevalence rate among Japanese patients since 1950 [14]. Therefore, SARS-CoV-2 infection could affect the clinical course of IBD patients, in whom intestinal inflammation is central to the pathogenesis and who require immunosuppressive therapy.

There are differences in the severity of SARS-CoV-2 infection and the prognosis of COVID-19 patients between other countries and Japan. The genetic background of IBD is also different. Thus, it is essential to capture the clinical characteristics of Japanese IBD patients with COVID-19 for the future diagnostic and medical interventions. Therefore, we established the registry cohort of Japanese IBD patients with COVID-19 to determine the incidence of the disease and the impact of COVID-19 on the clinical course of IBD. This study was referred to as Japan COVID19 surveillance in inflammatory bowel disease (JCOSMOS).

\section{Methods}

This is a multicenter, registry cohort study conducted by the research study group on intractable inflammatory bowel disorder of the Ministry of Health, Labor and Welfare in Japan. The study has been conducted using information obtained from eligible patients. No new intervention was implemented for this study. The protocol of this study was approved by the IRB at each institution and registered publicly on the University Hospital Medical Information Network registration number UMIN000040656.

\section{Patients}

Patients who were diagnosed with the following IBD types, such as (ulcerative colitis (UC), Crohn's disease (CD), Inflammatory bowel disease unclassified (IBDU), intestinal Behçet's disease (BD) and simple ulcer (SU), and who tested positive for COVID-19 were been eligible in the outpatient clinic or the participating institutions from January 1, 2020. A confirmed diagnosis of COVID-19 was defined as the presence of the SARS-CoV-2 genome as confirmed by real-time polymerase chain reaction (RTPCR) or the positivity of the antigen test of SARS-CoV-2 via nasopharyngeal swab or saliva and the positivity of antibodies against SARS-CoV-2 in serum [15-17]. Asymptomatic SARS-CoV-2 carriers were also included. We registered IBD patients who have completed hospitalization or have undergone outpatient treatment for COVID19 and excluded patients who have refused to participate in this study.

\section{Survey method}

The physicians conducted the survey using the medical records at each institution and entered the obtained information into an Excel file "Case Report Form" and provided the password-locked and anonymized "Case Report Form" to the person in charge at the Department of Gastroenterology, Sapporo Medical University by e-mail. An electronic data capture (EDC) system for clinical information registration is currently used since June 2021.

\section{Survey items}

Medical history: age, gender, height, weight, IBD diagnosis, smoking status, comorbidities (cardiovascular disease, diabetes, asthma, chronic respiratory disease, hypertension, malignancy, cerebrovascular disease, chronic renal disease, chronic liver disease, and others). Disease activity ("active" was defined as a partial Mayo score $\geqq 3$ with a rectal bleeding subscore $\geqq 1$ for UC [18, 19] and a HarveyBradshaw Index (HBI) $\geqq 5$ for CD [20], a subjective judgment of the attending physician for IBD-U, intestinal $\mathrm{BD}$, and $\mathrm{SU}$ ), duration of disease, disease type, treatment (5-aminosalicylic acid, thiopurines, steroids, calcineurin inhibitors, biologics, JAK inhibitors, nutritional therapy, cytapheresis), exacerbation of IBD, and changes in IBD treatment during COVID-19. Information on COVID-19: date of diagnosis, number of days from onset to diagnosis, testing methods that led to diagnosis (PCR, antibody, other), signs and symptoms of COVID-19 (fever, cough, dyspnea, pharyngitis, diarrhea, arthralgia-myalgia/asthenia, rhinitis, dysosmia, dysgeusia and dysphonia), presence of pneumonia, COVID-19 treatment and severity/outcomes (outpatient treatment, inpatient treatment, intensive care, death). We determined the severity of COVID-19 according to the WHO classification [21]. We defined an infection of a fully vaccinated person (at least dose of any vaccine) as a "vaccine breakthrough infection". To examine domestic vaccination coverage over time, we calculated the vaccination coverage among the older people aged 65 years and above and among all citizens including those aged 12 years and older who were eligible for vaccination, using vaccination records published by the Cabinet Secretariat Office, based on reports from the Vaccination Record System. 


\section{Statistical analysis}

Microsoft Excel 16.0 was used to record the patient's data and analyze the clinical background factors and results of disease. Analyses that require tests were performed using EZR software [22]. Nominal variables data were analyzed using Fisher's exact test and the odds ratio (OR) was calculated at $95 \%$ confidence intervals $(95 \% \mathrm{Cls})$. A t-test with $95 \% \mathrm{Cl}$ was performed to analyze the means data. The logistic regression analysis Stepwise method (AIC, backward/forward) was performed to analyze the relationship between COVID-19 severity and risk factors. A matplot was used to describe the line plot of repeated measurement data on the IBD-activity during COVID-19.

\section{Results}

\section{Patients' characteristics}

One hundred eighty-seven IBD patients with COVID-19 were registered between June 2020 and October 2021. The mean age was 42 , and the number of registered patients peaked at age 20-29 years (Fig. 1A). The diagnosis of patients was UC $(n=104), \mathrm{CD}(n=74), \operatorname{IBD}-\mathrm{U}(n=3)$, and $\operatorname{BD}(n=6)$. The number of females was $72(38.5 \%)$
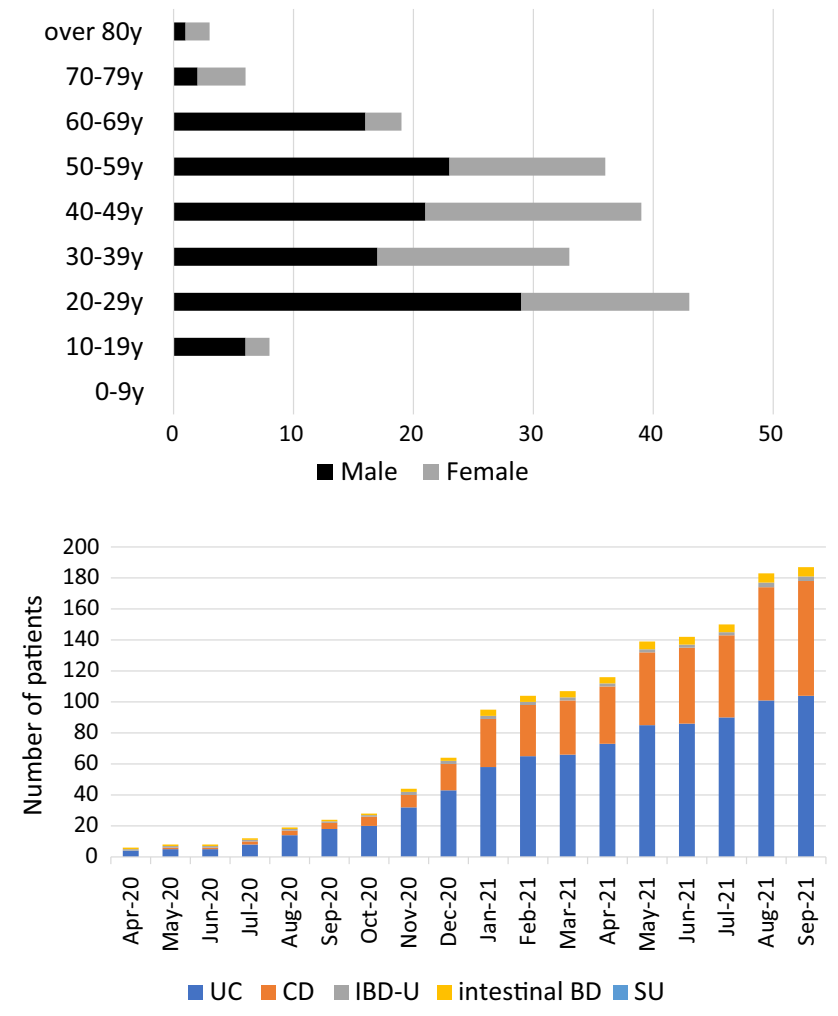

Fig. 1 (A) Age distribution of Japanese IBD patients with COVID19. (B) Transition of the number of Japanese IBD with COVID-19 and $30 \%$ of registered patients had existing comorbidities. The disease activity at the diagnosis of COVID-19 was $73 \%$ (136/187) for clinical remission, 22\% (42/187) for mildly active, $2.7 \%$ (5/187) for moderately active, and $2.1 \%$ (4/187) for severely active. Baseline characteristics of the patients are shown in Table 1 . We calculated the total number of patients by directly asking each facility with a patient registry about the number of IBD patients attending their hospitals. Based on that the total number of patients with IBD in 72 participating facilities, which was approximately 30,500, the estimated incidence of COVID19 in IBD patients was $0.61 \%$.

\section{Transition of the number of IBD patients with COVID-19 in Japan and vaccinated population}

The number of IBD patients with COVID-19 in Japan gradually increased and the upward trend was observed during the fifth wave of the pandemic (from August 2021 to September 2021) (Fig. 1B). In Japan, vaccine distribution for senior citizens started in April 2021. The first dose vaccination rate was $80 \%$ at the end of June, and the second dose vaccination rate exceeded $80 \%$ by the end of July, indicating that a large number of people have been vaccinated in a short period of time (Fig. 2A). After the start of the vaccination where the older people were prioritized, there was no significant increase in the number of registered patients over 60 years old. Meanwhile, the number of

Table 1 The characteristics of IBD patients with COVID-19

\begin{tabular}{ll}
\hline Patients' profile & \\
Age (years) \pm SD & $42.0 \pm 15.6$ \\
Gender (M/F) (n) & $115 / 72$ \\
Height $(\mathrm{cm}) \pm \mathrm{SD}$ & $166.0 \pm 9.4$ \\
Weight $(\mathrm{kg}) \pm \mathrm{SD}$ & $61.0 \pm 12.4$ \\
$\mathrm{BMI} \pm \mathrm{SD}$ & $21.6 \pm 4.4$ \\
BMI $>30(\mathrm{n})$ & 9 \\
Smoker (current) (\%) & $13 / 187(7.0 \%)$ \\
All comorbidity (\%) & $58 / 187(31.0 \%)$ \\
Diagnosis of IBD & \\
UC & 104 \\
CD & 74 \\
IBD-U & 3 \\
Intestinal BD & 6 \\
SU & 0 \\
Disease activity (at diagnosis of COVID-19) & \\
Remission & 136 \\
Mild & 42 \\
Moderate & 5 \\
Severe & 4
\end{tabular}



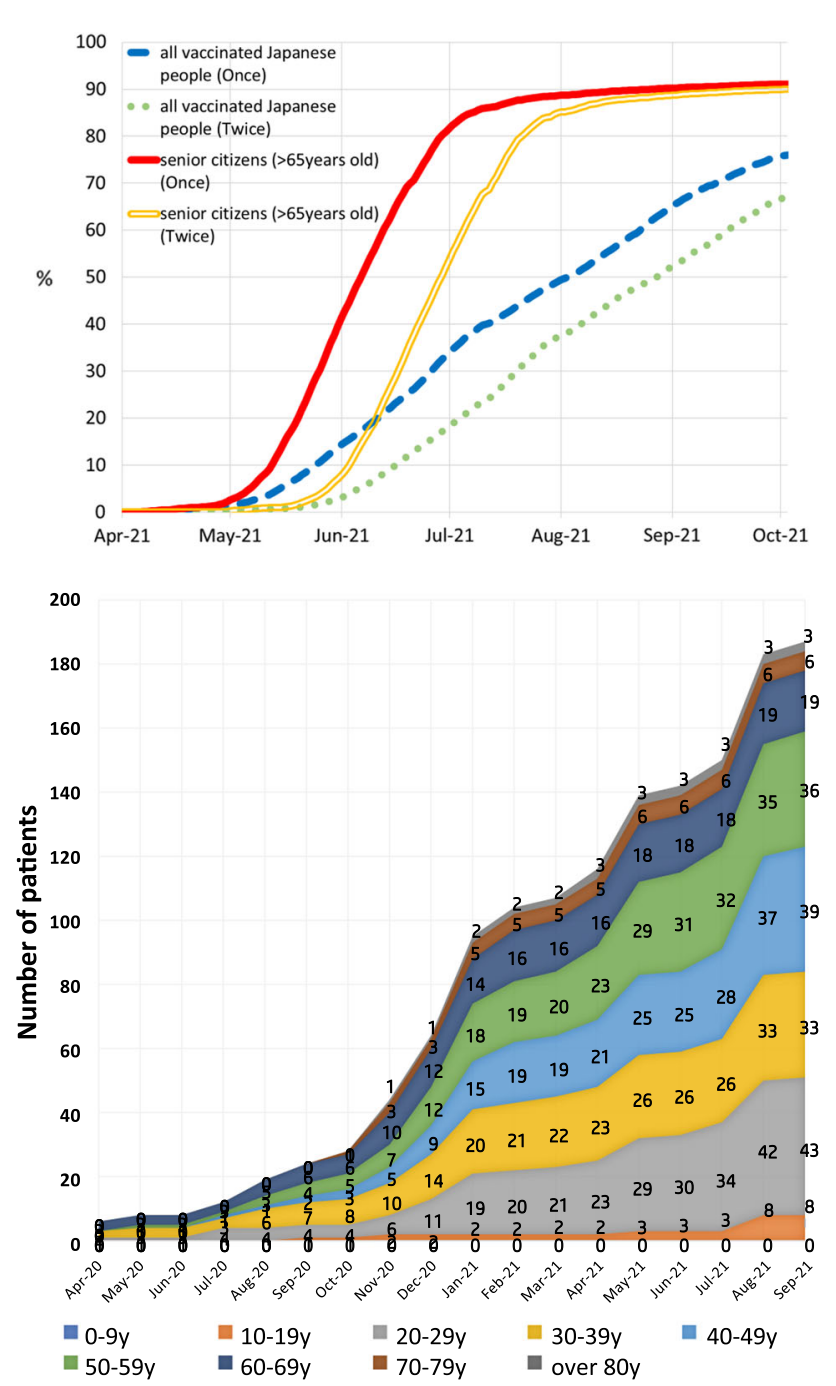

Fig. 2 (A) SARS-CoV-2 vaccination coverage (\%) in Japanese population. Vaccination coverage was calculated among all citizens who were eligible for vaccination, using vaccination records published by the Cabinet Secretariat Office, based on reports from the Vaccination Record System. (B) Transition of the number of Japanese IBD with COVID-19 stratified by age

patients in the age group of 20-50 years increased (Fig. 2B). The analyses of IBD patients with COVID-19 categorized according to region in Japan showed that Kanto district had the highest number of registered patients, followed by Hokkaido and Kansai districts (Supplementary Fig. 1). This is consistent to the trend that large cities tend to have a high numbers of COVID-19 cases $[23,24]$.

\section{IBD treatment}

Of the 187 registered patients, 147 were on 5-ASA, 75 on anti-TNF $\alpha$ antibodies, 58 on thiopurines, eight on steroids, and six on budesonide (Fig. 3). After the diagnosis of COVID-19, 52\% (30/58) and 36\% (27/75) of patients taking thiopurines and anti-TNF $\alpha$ antibodies, respectively, discontinued the medication.

\section{Symptoms during COVID-19}

The most common COVID-19 symptoms were fever $(75 \%)$, general fatigue $(52 \%)$, respiratory symptoms including cough (48\%), dysosmia/dysgeusia (32\%/33\%), and headache (24\%). A total of $23 \%$ (37/160) of COVID19 cases were related to pneumonia.

\section{COVD-19 severity and clinical outcome}

According to the WHO classification of COVID-19 severity, 93\% (172/184) of IBD patients had non-severe disease was, while $7 \%(12 / 184)$ of patients had severe disease. Of the 12 patients with severe COVID-19, 10 patients required oxygen and the remaining two required intensive care unit management and ventilation. No IBD patients died from COVID-19. The mean age of IBD patients with severe COVID-19 was significantly higher than that of the non-severe patients $(59.3 \pm 11.9$ vs. $40.7 \pm 15.2, \mathrm{p}<0.00005)$. In addition, the body mass index (BMI) [IQR] of IBD patients with severe COVID-19 was significantly higher than that of the non-severe patients (24.2 [23.1-29.2] vs. 21.5 [19.3-26.6], $\mathrm{p}=0.00467)$. IBD patients with severe COVID-19 had significantly higher rates of any comorbidity than those with non-severe COVID-19 (OR = 7.87). Among the comorbidities, Fisher's exact test showed there are significant differences among any respiratory illness $(\mathrm{OR}=16.3)$, hypertension $(\mathrm{OR}=8.8)$, and cerebrovascular disease $(\mathrm{OR}=16.3)$ between patients with severe COVID-19 and non-severe COVID-19 (Table 2). The rates of severe COVID-19 in patients with UC and CD were 11\% (11/101) and 1.3\% (1/ $74)$, respectively, although there was no statistically significant difference due to the small number of cases. In both UC and CD, neither the clinical phenotype nor disease activity was associated with COVID-19 severity. In the treatment of IBD, corticosteroids were associated with COVID-19 severity $(\mathrm{OR}=5.37)$, but not budesonide (Table 3).

Changes in IBD activity before and after COVID-19 were evaluated by pMayo score in UC and by HBI in CD. We found that $90.9 \%(165 / 187)$ of IBD patients with COVID-19 had no change in IBD disease activity. (Supplementary Fig. 2). Regarding the association between COVID-19 severity and risk factors, a logistic regression analysis stepwise method revealed that older age $(\mathrm{OR}=$ 1.07), high BMI $(\mathrm{OR}=1.18)$, and the steroid use $(\mathrm{OR}=$ 1.74) were risk factors for COVID-19, but anti-TNF $\alpha$ antibodies was not (Table 4, Supplementary Fig. 3, and 4). 
Fig. 3 Treatment of Japanese IBD at the diagnosis of COVID19. A blue bar indicated treatment continuation, an orange bar indicated treatment discontinuation, and a gray bar indicated additional treatment

Table 2 Clinical factors associated with COVID-19 severity in Japanese IBD patients

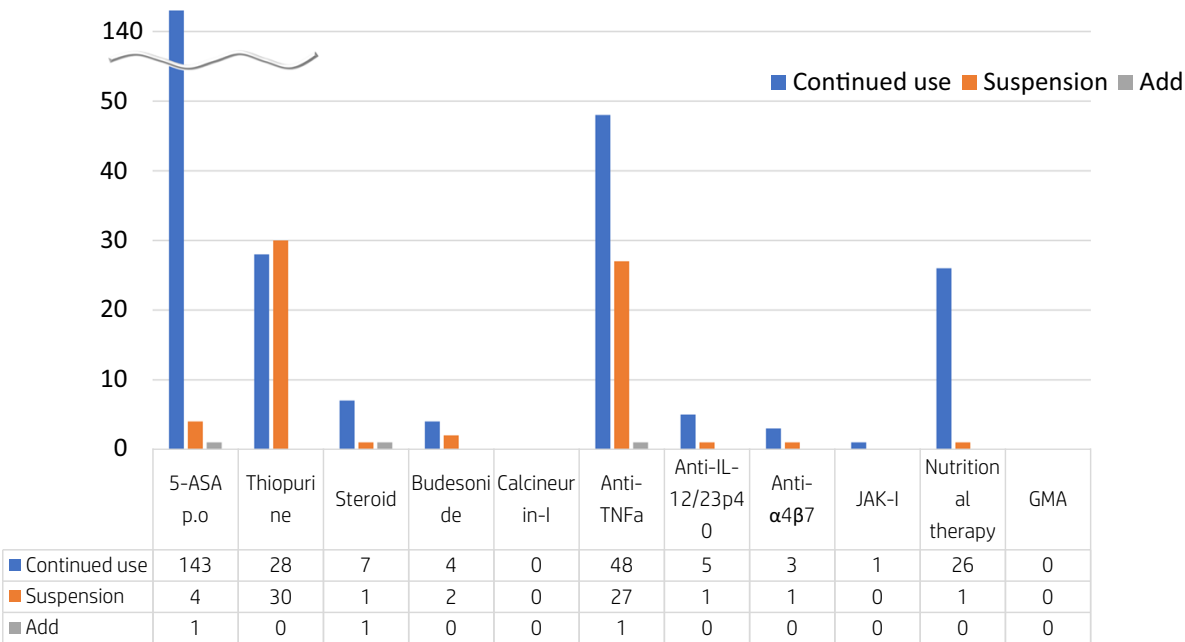

\begin{tabular}{lllll}
\hline Factor & Non-severe & Severe & Fisher-test $p$-value & Fisher-test odds \\
\hline Age \pm SD & $40.7 \pm 15.2$ & $59.3 \pm 11.9$ & 0.00005 & - \\
Gender M/F & $107 / 65$ & $7 / 5$ & 0.76800 & 1.17 \\
BMI [IQR] & $21.5[19.3-26.6]$ & $24.2[23.1-29.2]$ & 0.00467 & - \\
Comorbidity & & & & \\
DM & $4 / 172$ & $1 / 12$ & 0.28900 & 3.80 \\
CKD & $4 / 172$ & $0 / 12$ & 1.00000 & 0.00 \\
Liver diseases & $8 / 171$ & $0 / 12$ & 1.00000 & 0.00 \\
Asthma & $7 / 171$ & $2 / 12$ & 0.11000 & 4.60 \\
COPD & $0 / 172$ & $0 / 12$ & - & - \\
Other respiratory illness & $2 / 172$ & $2 / 12$ & 0.02180 & 16.30 \\
Cardiovascular diseases & $4 / 172$ & $0 / 12$ & 1.00000 & 0.00 \\
HT & $9 / 172$ & $4 / 12$ & 0.00551 & 8.80 \\
Cerebrovascular diseases & $2 / 172$ & $2 / 12$ & 0.02180 & 16.30 \\
Malignancy & $2 / 172$ & $0 / 12$ & 1.00000 & 0.00 \\
All & $47 / 172$ & $9 / 12$ & 0.00133 & 7.87 \\
Smoking & & & & - \\
Never & 121 & 6 & 0.11700 & \\
Current & 12 & 1 & & \\
P ast smoking & 29 & 5 & & \\
\hline
\end{tabular}

\section{COVID-19 cases after vaccination}

Nine IBD patients were recorded to have acquired COVID19 after vaccination. Five patients have CD, and four have UC, and neither had non-severe COVID-19. Three of them were considered breakthrough infections as more than 2 weeks have already passed since their second dose of vaccine. Six of the nine patients were receiving anti-TNF $\alpha$ antibodies, and four were receiving thiopurine. There was no progression of disease activity in nine IBD patients with COVID-19 despite the discontinuation of IBD drugs (Table 5).

\section{Discussion}

This is the first survey involving the characteristics and outcome of COVID-19 in IBD patients in Japan. Based on this survey, the estimated incidence of COVID-19 in patients with IBD is $0.61 \%$, which was lower than the current incidence of COVID-19 in the general population in Japan. The incident of COVID-19 was found higher in male than in female. Fever, general fatigue, and cough were the most common COVID-19 symptoms. Regarding the disease activity and IBD medication, most of the patients diagnosed with COVID-19 were in remission and the majority had been treated with 5-ASA alone, followed 
Table 3 IBD phenotype and treatments associated with COVID-19 severity in Japanese IBD patients

\begin{tabular}{|c|c|c|c|c|c|}
\hline Factor & & Non-severe & Severe & Fisher-test $p$-value & Fisher-test odds \\
\hline \multirow[t]{5}{*}{ Diagnosis of IBD } & UC & 90 & 11 & 0.06480 & - \\
\hline & $\mathrm{CD}$ & 73 & 1 & & \\
\hline & IBD-U & 3 & 0 & & \\
\hline & $\mathrm{BD}$ & 6 & 0 & & \\
\hline & SU & 0 & 0 & & \\
\hline \multicolumn{6}{|l|}{ Clinical phenotype } \\
\hline \multirow[t]{4}{*}{ UC } & Proctitis & 14 & 1 & 0.93200 & - \\
\hline & Left side & 28 & 4 & & \\
\hline & Pancolitis & 45 & 5 & & \\
\hline & segmental & 3 & 0 & & \\
\hline \multirow[t]{4}{*}{$\mathrm{CD}$} & Ileitis & 18 & 0 & 0.93200 & - \\
\hline & Colitis & 15 & 0 & & \\
\hline & Ileo-colitis & 42 & 1 & & \\
\hline & Isolated upper & 14 & 1 & & \\
\hline \multirow[t]{4}{*}{ Disease activity (at diagnosis of COVID-19) } & Remission & 124 & 10 & 0.24600 & - \\
\hline & Mild & 40 & 1 & & \\
\hline & Moderate & 5 & 0 & & \\
\hline & Severe & 3 & 1 & & \\
\hline \multirow[t]{9}{*}{ IBD treatment } & 5-ASA p.o & $132 / 170$ & $12 / 12$ & 0.07430 & Inf \\
\hline & Thiopurine & $55 / 172$ & $2 / 12$ & 0.34700 & 0.43 \\
\hline & Steroid & $6 / 170$ & $2 / 12$ & 0.08950 & 5.37 \\
\hline & Budesonide & $6 / 172$ & $0 / 12$ & 1.00000 & 0.00 \\
\hline & Calcineurin-inhibitors & $0 / 172$ & $0 / 12$ & - & - \\
\hline & Anti-TNF $\alpha$ antibodies & $73 / 171$ & $1 / 12$ & 0.02920 & 0.12 \\
\hline & Anti-IL-12/23p40 & $6 / 172$ & $0 / 12$ & 1.00000 & 0.00 \\
\hline & Anti- $\alpha 4 \beta 7$ & $4 / 172$ & $0 / 12$ & 1.00000 & 0.00 \\
\hline & JAK-inhibitor & $1 / 170$ & $0 / 12$ & 1.00000 & 0.00 \\
\hline
\end{tabular}

Missing values: three UC patients whose severity of COVID-19 according to WHO classification was not known

Table 4 Risk factor of COVID-19 severity in Japanese IBD patients

\begin{tabular}{lll}
\hline Risk factor & Odds ratio & $p$ value \\
\hline Age & 1.07 & 0.0101 \\
BMI & 1.18 & 0.00812 \\
5-ASA & $1.34 \mathrm{E}+07$ & 0.992 \\
Steroid & 1.74 & 0.0218 \\
\hline
\end{tabular}

Logistic regression $\mathrm{AIC}=66.39$

Method: logistic regression analysis Stepwise method (AIC, backward/forward)

Objective variable: COVID-19 severity in the WHO classification

Explanatory variables: age, presence of comorbidities, BMI, IBD diagnosis, and IBD treatment

by anti-TNF- $\alpha$ antibodies. In this current survey, the registered number of Japanese IBD patients with COVID-19 receiving steroids was low. A logistic regression analysis stepwise method revealed that older age, higher BMI, and steroid use were risk factors for COVID-19 severity. Overall, our findings confirmed that the general risk factors for severe outcomes of COVID-19 in IBD patients are similar to other studies of IBD patients.

The number of Japanese IBD patients with COVID-19 gradually increased until the fifth wave of COVID-19, however, also coincided with the largest increase of COVID-19 cases in the Japanese general population. One of the reasons for the increase in IBD patients with COVID-19 on the fifth wave is associated with the spread of SARS-CoV-2 infections in younger people, which at that time remained unvaccinated. Meanwhile, it should be noted that there was no increase in the number of older IBD patients registered during the fifth wave since the senior citizens had been priority for vaccination. The current incidence rate of COVID-19 in Japan (1.73 million/126.1 


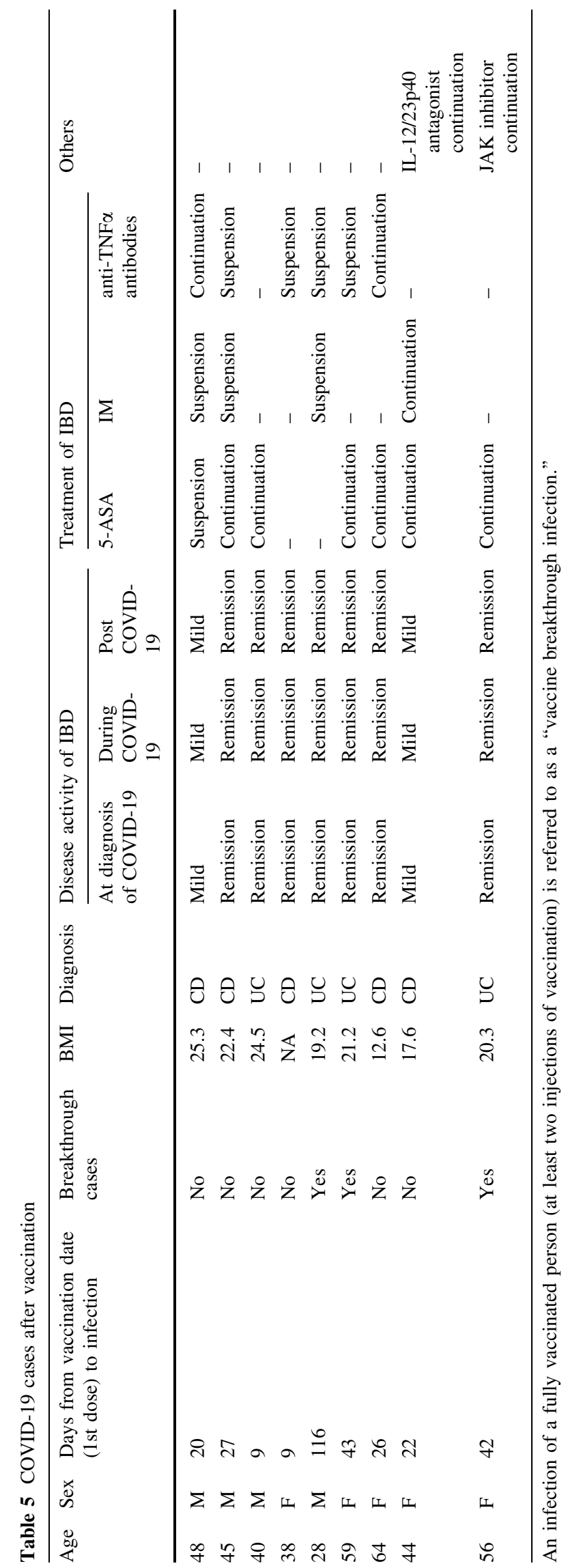


million people) is $1.37 \%$ [25]. In this cohort, the estimated incidence rate which we calculated based on the number of IBD patients registered by the participating facilities is $0.61 \%$. This can be attributed to the fact that most of the Japanese patients with IBD often pay attention to the risk of an infection, not limited to SARS-CoV-2, and that they are particularly observing social distancing during the pandemic.

In this survey, the percentage of Japanese IBD patient with severe COVID-19 based on WHO classification was $7 \%$ and with no cases of death recorded. Surveillance Epidemiology of Coronavirus under Research Exclusion (SECURE-IBD) data showed the primary outcome (ICU/ ventilation/death) was observed in 37/525 (7\%) of patients, and with 3\% death of reported cases [26]. Systematic review on IBD patients with COVID-19 by D' Amico showed that 28/246 (11.4\%) of patients stayed in ICU, 26/697 (3.7\%) of patients needed for mechanical ventilation, and 29/760 (3.8\%) of patients died [27]. These previous data are not directly comparable to our survey data in the frequency of COVID-19 severity because of the lack of severity assessment according to the WHO classification in other studies. However, it is noteworthy that there have been no deaths among Japanese IBD patient with COVID19 up to this time. Given that about a half of patients enrolled in the cohort were on biologics and the number of patients used corticosteroids was low, the use of anti-TNF $\alpha$ antibodies may contribute to the lower rate of severe COVID-19, with no death cases. Several reports indicates that anti-TNF $\alpha$ antibodies and JAK inhibitors could regulate the pro-inflammatory cytokine production caused by COVID-19 infection [28, 29]. Further, recent clinical trial supported the suppression of COVID-19 severity by JAK inhibitors [30]. This cohort has not yet enrolled many patients on JAK inhibitors or biologics other than anti$\mathrm{TNF} \alpha$ antibodies, therefore, further survey is required.

Multivariable analysis of SECURE-IBD data demonstrated that increasing age, more than two comorbidities, systemic corticosteroid, and 5-ASA/sulfasalazine used were positively associated with adverse COVID-19 outcomes in IBD patients and the use of immunomodulatory therapy including anti-TNF $\alpha$ antibodies was not associated with risk of morbidity [31]. We found older age, BMI, and presence of comorbidities, and the use of corticosteroids as factors associated with increased severity, was supported by the SECURE-IBD data. Despite the small number of patients on steroids, it should be noted that steroids were still extracted as a risk factor for severe COVID-19.

In this registry, we found that the rate of severe COVID19 in patients with UC was higher than CD. The possible reasons of the difference in the rate of severity of COVID19 between patients with UC and CD are (1) the majority of registered patients over 60 are patients with UC
(Supplementary Fig. 5) and (2) the usage rate of anti-TNFa antibodies in UC and CD patients at the diagnosis of COVID-19 was $18.2 \%$ (19/104) and $70.2 \%$ (52/74), respectively (Supplementary Table 1). These factors might contribute to the higher proportion of severe COVID-19 in $\mathrm{UC}$ than $\mathrm{CD}$.

Regarding the change of disease activity in IBD patients with COVID-19, the results of this survey showed that COVID-19 did not affect disease activity in most IBD patients with COVID-19, except for a few IBD patients who had exacerbations. Luki and colleagues reported that COVID-19 has no durable impact of on intestinal disease of IBD patients based on change of clinical activity scores and endoscopic scores on COVID-19 positive IBD patients despite increasing the level of fecal calprotectin (fCal) at the course of COVID-19 [32]. The change of fCal without exacerbating abdominal symptoms may reflect the involvement of SARS-CoV-2 in the mild intestinal inflammation via ACE2 receptor [33], although we could not observe the change of fecal calprotectin in our survey.

Post-vaccination COVID-19 is one of the clinical challenges in COVID-19 management [34]. This survey showed nine patients had COVID-19 after first or second vaccination, three of them were considered breakthrough infections, and the others had COVID-19 before the second dose. All patients had non-severe COVID-19. Six of seven patients had received anti-TNF $\alpha$ antibodies and there was no worsening of disease activity in seven IBD patients with COVID-19 post vaccination despite the discontinuation of IBD drugs. A recent report indicated that anti-TNF $\alpha$ antibodies could affect anti-SARS-CoV-2 antibody concentrations [35, 36]. However, the association between the use of anti-TNF $\alpha$ antibodies and the onset of COVID-19 after vaccination remains unclear in the current survey because we could not measure antibodies against SARS-CoV-2 at this time. A study on antibody titer after COVID-19 vaccination in Japanese IBD patients is underway, and the result would illuminate how IBD drugs affect SARS-CoV2 antibody concentrations after vaccination.

Our study has several limitations. First, not all IBD patients with COVID-19 in Japan were included because of lack of national registry for IBD patients including pediatric patients. However, the data is reliable because all patients were registered from facilities with IBD specialists. Second, the incidence of COVID-19 after two doses of vaccination has not yet been determined, and it is important to continue the registry in the future. Third, in the present survey, we did not collect data regarding the types of antiTNF- $\alpha$ antibody agent in detail. The rate of discontinuation of anti-TNF- $\alpha$ antibody agents was high after patients had COVID-19; however, we should consider that differences in administration intervals may have influenced the decision of the discontinuation of the agents. 
In conclusion, we first reported the results of the interim analysis of the COVID-19 registry in Japanese IBD patients. The incidence of COVID-19 in Japanese IBD patients was found to be lower than the general population. Older age and the presence of comorbidities were factors in the incidence of contacting COVID-19, which was the same trend as in the general population. Various factors related to the risk and severity of COVID-19 may be extracted based on accumulation of COVID-19 cases in the future. Further, follow-up study is necessary to confirm our current results.

Supplementary InformationThe online version contains supplementary material available at https://doi.org/10.1007/s00535022-01851-1.

Acknowledgements Toshiki Ichimori (Susaki Kuroshio Hospital), Reikei Matsuda (Fujisawa Shounandai Hospital), Tadashi Yokoyama (Yokoyama IBD Clinic). Members of the J-COSMOS group (name and affiliation) are Makoto Sasaki (Aichi Medical University), Masahiro Iizuka (Akita Red Cross Hospital), Mikihiro Fujiya (Asahikawa Medical University), Takayuki Matsumoto (Iwate Medical University), Fukunori Kinjo (Urasoe General Hospital), Shiro Nakamura (Osaka Medical and Pharmaceutical University), Noriko Kamata (Osaka City University Hospital), Hideki Iijima (Graduate School of Medicine Faculty of Medicine, Osaka University), Yuri Etani (Osaka Women's and Children's Hospital), Fumiaki Ueno (Ofuna Chuo Hospital), Sakiko Hiraoka (Okayama University Hospital), Takeo Kondo (Kagawa University Hospital), Takashi Kagaya (National Hospital Organization Kanazawa Medical Center), Makoto Naganuma (Kansai Medical University), Kiyonori Kobayashi (Kitasato University School of Medicine), Taku Kobayashi (Kitasato University Kitasato Institute Hospital), Shuji Yamamoto (Graduate School of Medicine and Faculty of Medicine Kyoto University), Yuji Naito (Kyoto Prefectural University of Medicine), Tadakazu Hisamatsu (Kyorin University School of Medicine), Yoki Furuta (Kumamoto University Hospital), Keichi Mitsuyama (Kurume University school of Medicine), Yu Hashimoto (Gunma University Hospital), Katsuhiro Arai (National Center for Child Health and Development), Shingo Kato (Saitama Medical Center), Itaru Iwama (Saitama Prefectual Children's Medical Center), Motohiro Esaki (Saga University Hospital), Hiroki Tanaka (Sapporo IBD Clinic), Hiroshi Nakase (Sapporo Medical University School of Medicine), Satoshi Motoya (Sapporo-Kosei General Hospital), Atsuo Maemoto (Sapporo Higashi Tokushukai Hospital), Tomofumi Ashida (Sapporo Tokushukai Hospital), Nobuaki Nishimata (Sameshima Hospital), Akira Andoh (Shiga University of Medical Science), Hironori Yamamoto (Jichi Medical University), Shunji Ishihara (Faculty of Medicine Shimane University), Toshiaki Shimizu (Juntendo University Hospital), Yasuharu Maeda (Showa University Northern Yokohama Hospital), Kenji Kinoshita (Hakodate Municipal Hospital), Katuyuki Fukuda (St. Luke's International Hospital), Jun Kato (Chiba University Hospital), Ken Takeuchi (Tsujinaka Hospital Kashiwanoha), Masakazu Nagahori (Tokyo Medical and Dental University), Masakatsu Fukuzawa (Tokyo Medical University), Masayuki Saruta (The Jikei University School of Medicine), Michio Itabashi (Tokyo Women's Medical University), Masaru Shinozaki (Research Hospital The Institute of Medical Science The University of Tokyo), Soichiro Ishihara (Faculty of Medicine The University of Tokyo), Naoki Yoshimura (Tokyo Yamate Medical Center), Katsuyoshi Matsuoka (Toho University Sakura Medical Center), Yoichi Kakuta (Department of Gastroenterology, Tohoku University), Kenichi Takahashi (Tohoku Rosai Hospital), Ryosuke Sakemi (Tobata
Kyoritsu Hospital), Sohachi Nanjo (Toyama University Hospital), Shusaku Yoshikawa (Dongo Hospital), Keiji Ozeki (Nagoya City University Graduate School of Medical), Ayako Fuchigami (Nippon Koukan Hospital), Takehiko Katsurada (Hokkaido University Faculty of Medicine and Graduate School of Medicine), Kenji Watanabe (The Hospital of Hyogo College of Medicine), Hirotake Sakuraba (School of Medicine Hirosaki University), Fumihito Hirai (Fukuoka University Faculty of Medicine), Takashi Hisabe (Fukuoka University Chikushi Hospital), Naoki Ohmiya (FUJITA HEALTH UNIVERSITY HOSPITAL), Ryota Hokari (National Defence Medical College Hospital), Katsuhiko Nakai (Matsuda Hospital), Daiki Abukawa (Miyagi Children's Hospital), Shojiro Yamamoto (University of Miyazaki Hospital), Kazutaka Koganei (Yokohama Municipal Citizen's Hospital), Reiko Kunisaki (Yokohama City University Medical Center), Akira Hokama (University of the Ryukyus Hospital).

Author contributions All author contributions to the manuscript are as follows: drafting of the manuscript (HN, YH, DH, TM, MM, HI, $\mathrm{KM}, \mathrm{NO}, \mathrm{SI}, \mathrm{FH}, \mathrm{DA}$, and TH), data collection and data analysis (HN, $\mathrm{YH}, \mathrm{DH})$, critical revision of the manuscript for important intellectual content (HN, YH, DH, TM, MM, HI, KM, NO, SI, FH, DA, and TH).

Funding This work was supported by Health and Labour Sciences Research Grants for research on intractable diseases from the Ministry of Health, Labour and Welfare of Japan. (Investigation and Research for intractable Inflammatory Bowel Disease).

\section{Declarations}

Conflict of interest Dr. Hiroshi Nakase (HN) reports receiving personal fees from Abbvie Inc., Kissei Pharmaceutical Co., Ltd., KYORIN Pharmaceutical Co., Ltd, Mitsubishi Tanabe Pharma Corporation, Janssen Pharmaceutical K.K, Takeda Pharmaceutical Co., Ltd., Pfizer Japan Inc., Celgene K.K., EA Pharma Co., Ltd., Zeria Pharmaceutical CO., Ltd., Mochida Pharmaceutical Co., Ltd., Nippon Kayaku Co., Ltd., D Daiichi Sankyo Co., Ltd., JIMRO Co., Ltd., as well as grants for commissioned/joint research from Hoya Group Pentax Medical, Boehringer Ingelheim GmbH, Bristol-Myers Squibb Company. Dr Yuki Hayashi (YH) and Dr. Daisuke Hirayama (DH) report no receipt of personal fees from any pharmaceutical companies. Dr. Takayuki Matsumoto (TM) reports receipt of personal fees from Abbvie Inc., Kissei Pharmaceutical Co., Ltd., Kyorin Pharmaceutical Co., Ltd., Janssen Pharmaceutical K.K, Takeda Pharmaceutical Co., Ltd., EA Pharma Co., Ltd., Mochida Pharmaceutical Co., Ltd., Nippon Kayaku Co., Ltd., Mitsubishi Tanabe Pharma Corporation, and JIMRO Co., Ltd. Dr. Minoru Matsuura (MM) reports receipt of personal fees from AbbVie GK, Mitsubishi Tanabe Pharma Corporation, Nippon Kayaku Co., Ltd., Janssen Pharmaceutical K.K., Takeda Pharmaceutical Co. Ltd., Kyorin Pharmaceutical Co., Ltd., and Mochida Pharmaceutical Co., Ltd. Dr. Hideki Iijima (HI) report personal fees from AbbVie Inc, Janssen Pharmaceutical K.K., EA Pharma Co., Ltd., Eisai Pharmaceutical Co., Ltd., Mitsubishi Tanabe Pharma Co., Ltd., Mochida Pharmaceutical Co. Ltd., and research grants from AbbVie Inc., Nippon Kayaku Co., Ltd, Daiichi Sankyo Company, Ltd. Dr. Katsuyoshi Matsuoka (KM) reports personal fees from AbbVie Inc, Kissei Pharmaceutical Co., Ltd., Mitsubishi Tanabe Pharma Co., Ltd., Janssen Pharmaceutical K.K., Takeda Pharmaceutical Co., Ltd., Pfizer Lnc., EA Pharma Co., Ltd., Mochida Pharmaceutical Co., Ltd., and commercial research funding from Mitsubishi Tanabe Pharma Corporation, Mochida Pharmaceutical Co., Ltd, AbbVie GK, Nippon Kayaku Co., Ltd., EA pharma Co., Ltd. Dr. Naoki Ohmiya (NO) reports receiving grants for commissioned/ joint research from Nippon Kayaku Co., Ltd. EA Pharma Co., Ltd., EA Pharma Co., Ltd. Dr. Shunji Ishihara (SI) reports receipt of personal fees from Takeda Pharmaceutical Co. Ltd and receiving grants 
for commissioned/joint research from Takeda Pharmaceutical Co. Ltd, Nippon Kayaku Co., Ltd., Astellas Pharma Inc., EA Pharma Co., Ltd., Zeria Pharmaceutical CO., Ltd., Daiichi Sankyo Company, Ltd., and JIMRO Co., Ltd. Dr. Fumihito Hirai (FH) reports receipt of personal fees from AbbVie GK, EA pharma Co., Ltd., Janssen Pharmaceutical K.K., Mochida Pharmaceutical Co., Ltd., Mitsubishi Tanabe Pharma Corporation, Takeda Pharmaceutical Co. Ltd. Dr. Daiki Abukawa reports no receipt of personal fees from any pharmaceutical companies. Dr. Tadakazu Hisamatsu $(\mathrm{TH})$ receipt of personal fees from EA pharma Co. Ltd., AbbVie GK, Celgene K.K., Janssen Pharmaceutical K.K., Pfizer Inc., Mitsubishi Tanabe Pharma Corporation, Kyorin Pharmaceutical Co. Ltd., JIMRO Co., Ltd., Mochida Pharmaceutical Co., Ltd., Nichi-lko Pharmaceutical Co., Ltd., and commercial research funding from EA pharma Co., Ltd., AbbVie GK, Daiichi-Sankyo Co., Ltd., Takeda Pharmaceutical Co. Ltd., Pfizer Inc., Mochida Pharmaceutical Co., Ltd, Nippon Kayaku Co., Ltd., Kyorin Pharmaceutical Co., Ltd., JIMRO Co.,Ltd., Mochida Pharmaceutical Co., Ltd., and ZERIA Pharmaceutical Co., Ltd.

Open Access This article is licensed under a Creative Commons Attribution 4.0 International License, which permits use, sharing, adaptation, distribution and reproduction in any medium or format, as long as you give appropriate credit to the original author(s) and the source, provide a link to the Creative Commons licence, and indicate if changes were made. The images or other third party material in this article are included in the article's Creative Commons licence, unless indicated otherwise in a credit line to the material. If material is not included in the article's Creative Commons licence and your intended use is not permitted by statutory regulation or exceeds the permitted use, you will need to obtain permission directly from the copyright holder. To view a copy of this licence, visit http://creativecommons. org/licenses/by/4.0/.

\section{References}

1. Sungnak W, Huang N, Bécavin C, et al. SuSARS-CoV-2 entry factors are highly expressed in nasal epithelial cells together with innate immune genes. Nat Med. 2020;26:681-7.

2. Zhu N, Zhang D, Wang $\mathrm{W}$, et al. A novel coronavirus from patients with pneumonia in China, 2019. N Engl J Med. 2020;382:727-33.

3. World Health Organization. Naming the coronavirus disease (COVID-19) and the virus that causes it. https://www.who.int/ emergencies/diseases/novel-coronavirus-2019/technical-gui dance/naming-the-coronavirus-disease-(covid-2019)-and-thevirus-that-causes-it (2020).

4. Centers for Disease Control and Prevention (CDC). People Who Are at Higher Risk for Severe Illness. 2020. https://www.cdc. gov/coronavirus/2019-ncov/need-extra-precautions/people-athigher-risk.html.

5. Freites Nuñez DD, Leon L, Mucientes A, et al. Risk factors for hospital admissions related to COVID-19 in patients with autoimmune inflammatory rheumatic diseases. Ann Rheum Dis. 2020;79:1393-9.

6. Holman N, Knighton P, Kar P, et al. Risk factors for COVID-19related mortality in people with type 1 and type 2 diabetes in England: a population-based cohort study. Lancet Diabetes Endocrinol. 2020;8:823-33.

7. Chen N, Zhou M, Dong X, et al. Epidemiological and clinical characteristics of 99 cases of 2019 novel coronavirus pneumonia in Wuhan, China: a descriptive study. Lancet. 2020;395:507-13.
8. Wang D, Hu B, Hu C, et al. Clinical characteristics of 138 hospitalized patients with 2019 novel coronavirus-infected pneumonia in Wuhan. China JAMA. 2020;323:1061-9.

9. Tian Y, Rong L, Nian W, et al. Review article: gastrointestinal features in COVID-19 and the possibility of faecal transmission. Aliment Pharmacol Ther. 2020;51:843-51.

10. Sultan S, Lim JK, Atayar O, et al. AGA institute rapid recommendations for gastrointestinal procedures during the COVID-19 pandemic. Gastroenterology. 2020;159:739-58.e4.

11. Hayashi Y, Wagatsuma K, Nojima M, et al. The characteristics of gastrointestinal symptoms in patients with severe COVID-19: a systematic review and meta-analysis. J Gastroenterol. 2021;56:409-20.

12. Effenberger M, Grabherr F, Mayr L, et al. Faecal calprotectin indicates intestinal inflammation in COVID-19. Gut. 2020;69:1543-4.

13. Neurath MF. COVID-19 and immunomodulation in IBD. Gut. 2020;69:1335-42.

14. Nakase H, Uchino M, Shinzaki S, et al. Evidence-based clinical practice guidelines for inflammatory bowel disease 2020. J Gastroenterol. 2021;56:489-526.

15. Information for Laboratories about Coronavirus (COVID-19) https://www.cdc.gov/coronavirus/2019-ncov/lab/index.html

16. Coronavirus Disease 2019 Testing Basics https://www.fda.gov/ consumers/consumer-updates/coronavirus-disease-2019-testingbasics.

17. Wang YK, Kang H, Liu X, et al. Combination of RT-qPCR testing and clinical features for diagnosis of COVID-19 facilitates management of SARS-CoV-2 outbreak. J Med Virol. 2020;92:538-9.

18. Schroeder KW, Tremaine WJ, Ilstrup DM. Coated oral 5-aminosalicylic acid therapy for mildly to moderately active ulcerative colitis. A randomized study. $N$ Engl J Med. 1987; 317:1625-9.

19. D’Haens G, Sandborn WJ, Feagan BG, et al. A review of activity indices and efficacy end points for clinical trials of medical therapy in adults with ulcerative colitis. Gastroenterology. 2007;132:763-86.

20. Harvey RF, Bradshaw JM. A simple index of Crohn's-disease activity. Lancet. 1980;1:514.

21. COVID-19 Treatment Guidelines. Clinical spectrum of SARSCoV-2 infection. https://www.covid19treatmentguidelines.nih. gov/overview/clinical-spectrum/.

22. Kanda Y. Investigation of the freely available easy-to-use software 'EZR' for medical statistics. Bone Marrow Transplant. 2013;48:452-8.

23. Ni Z, Lebowitz ER, Zou Z, et al. Response to the COVID-19 outbreak in urban settings in China. J Urban Health. 2021;98:41-52.

24. Sharifi A, Khavarian-Garmsir AR. The COVID-19 pandemic: Impacts on cities and major lessons for urban planning, design, and management. Sci Total Environ. 2020; 749:142391.

25. https://www.mhlw.go.jp/stf/covid-19/kokunainohasseijoukyou. html.

26. Brenner EJ, Ungaro RC, Gearry RB, et al. Corticosteroids, but not TNF antagonists, are associated with adverse COVID-19 outcomes in patients with inflammatory bowel diseases: results from an international registry. Gastroenterology. 2020;59:48191.e3.

27. D'Amico F, Danese S, Peyrin-Biroulet L. Systematic review on inflammatory bowel disease patients with coronavirus disease 2019: it is time to take stock. Clin Gastroenterol Hepatol. 2020;18:2689-700.

28. Robinson PC, Richards D, Tanner HL, et al. Accumulating evidence suggests anti-TNF therapy needs to be given trial priority in COVID-19 treatment. Lancet Rheumatol. 2020;2:e653-5. 
29. Seif F, Aazami H, Khoshmirsafa M, et al. JAK inhibition as a new treatment strategy for patients with COVID-19. Int Arch Allergy Immunol. 2020;181:467-75.

30. Kalil AC, Patterson TF, Mehta AK, et al. Baricitinib plus remdesivir for hospitalized adults with Covid-19. N Engl J Med. 2021;384:795-807.

31. Izadi Z, Brenner EJ, Mahil SK, et al. Psoriasis Patient Registry for Outcomes, Therapy and Epidemiology of COVID-19 Infection (PsoProtect); the Secure Epidemiology of Coronavirus Under Research Exclusion for Inflammatory Bowel Disease (SECUREIBD); and the COVID-19 Global Rheumatology Allianc; Psoriasis Patient Registry for Outcomes, Therapy and Epidemiology of COVID-19 Infection (PsoProtect); the Secure Epidemiology of Coronavirus Under Research Exclusion for Inflammatory Bowel Disease (SECURE-IBD); and the COVID-19 Global Rheumatology Alliance (GRA). Association Between Tumor Necrosis Factor Inhibitors and the Risk of Hospitalization or Death Among Patients With Immune-Mediated Inflammatory Disease and COVID-19. JAMA Netw Open. 2021;4:e2129639.

32. Lukin DJ, Funez-dePagnier G, Lima S, et al. No Durable impact of COVID-19 on intestinal disease activity in subjects with IBD. Clin Gastroenterol Hepatol. 2021;19:2312-2314.e3.
33. Suárez-Fariñas $M$, Tokuyama $M$, Wei $G$, et al. Intestinal inflammation modulates the expression of ACE2 and TMPRSS2 and potentially overlaps with the pathogenesis of SARS-CoV-2related disease. Gastroenterology. 2021;160:287-301.e20.

34. Ben-Tov A, Banon T, Chodick G, et al. BNT162b2 messenger RNA COVID-19 vaccine effectiveness in patients with inflammatory bowel disease: preliminary real-world data during mass vaccination campaign. Gastroenterology. 2021;161:17151717.e1.

35. Kennedy NA, Goodhand JR, Bewshea C, et al. Anti-SARS-CoV2 antibody responses are attenuated in patients with IBD treated with infliximab. Gut. 2021;70:865-75.

36. Kennedy NA, Lin S, Goodhand JR, et al. Infliximab is associated with attenuated immunogenicity to BNT162b2 and ChAdOx1 nCoV-19 SARS-CoV-2 vaccines in patients with IBD. Gut. 2021;70:1884-93.

Publisher's Note Springer Nature remains neutral with regard to jurisdictional claims in published maps and institutional affiliations. 